\title{
BGO front-end electronics and signal processing in the MXGS instrument for the ASIM
} mission

Skogseide, Yngve; Cenkeramaddi, Linga Reddy; Genov, Georgi; Njoten, Kare; Rostad, Maja Elise; Solberg, Arne; Stadsnes, Johan; Ullaland, Kjetil; Ostgaard, Nikolai; Budtz-Jørgensen, Carl

Total number of authors:

11

Published in:

Proceedings of IEEE Instrumentation/Measurement Technology Conference (I2MTC)

Link to article, DOI:

10.1109/I2MTC.2012.6229186

Publication date:

2012

Document Version

Publisher's PDF, also known as Version of record

Link back to DTU Orbit

Citation $(A P A)$ :

Skogseide, Y., Cenkeramaddi, L. R., Genov, G., Njoten, K., Rostad, M. E., Solberg, A., Stadsnes, J., Ullaland, K., Ostgaard, N., Budtz-Jørgensen, C., \& Kuvvetli, I. (2012). BGO front-end electronics and signal processing in the MXGS instrument for the ASIM mission. In Proceedings of IEEE Instrumentation/Measurement Technology Conference (I2MTC) (pp. 2124-2127). IEEE. https://doi.org/10.1109//2MTC.2012.6229186

\section{General rights}

Copyright and moral rights for the publications made accessible in the public portal are retained by the authors and/or other copyright owners and it is a condition of accessing publications that users recognise and abide by the legal requirements associated with these rights.

- Users may download and print one copy of any publication from the public portal for the purpose of private study or research.

- You may not further distribute the material or use it for any profit-making activity or commercial gain

- You may freely distribute the URL identifying the publication in the public portal 


\section{BGO Front-End Electronics and Signal Processing in the MXGS Instrument for the ASIM Mission}

\author{
Yngve Skogseide, Linga Reddy Cenkeramaddi, Georgi \\ Genov, Kåre Njøten, Maja Elise Rostad, Arne Solberg, \\ Johan Stadsnes, Kjetil Ullaland, Nikolai Østgaard \\ University of Bergen \\ Department of Physics and Technology \\ Bergen, Norway
}

\begin{abstract}
This paper presents the Bismuth Germanate Oxide (BGO) front-end electronics design and signal processing in Modular X- and Gamma ray sensor (MXGS) instrument onboard the Atmosphere Space Interaction Monitor (ASIM) mission, funded by the European Space Agency. University of Bergen is responsible for the design and development of the detector layers and readout electronics for the MXGS instrument. The principal objective of the instrument is to detect Terrestrial Gamma ray Flashes (TGFs), which are related to thunderstorm activity.
\end{abstract}

The digital pulse processing scheme used in the MXGS BGO detector gives it a significantly higher rate capability than what has been achieved in other instruments used in the study of terrestrial gamma flashes. The front-end electronics for the BGO detector layer in MXGS system also uses fewer components compared to conventional analog front-ends for BGO detectors, thereby increasing its reliability and projected lifetime in the harsh space environment. The MXGS instrument is expected to see about 1000 TGFs in a time period of one year.

Index terms: Gamma ray detector, $X$-ray detetctor, .Radiation Imaging, BGO detector, International Space Station, MXGS instrument, ASIM mission, Terrestrial Gamma Flashes.

\section{INTRODUCTION}

The Atmosphere-Space Interaction Monitor (ASIM) payload is scheduled to be flown on the the Columbus External Platform Facility (CEPF) of the International Space Station (ISS), with a planned launch in 2014. The main objective of ASIM is to observe Terrestrial Gamma Flashes (TGFs) and Transient Luminous Events (TLEs), known as blue jets, sprites and elves and how these phenomena are related to thunderstorms and lightning discharges. TGFs were discovered in 1994 [1] as short flashes $(<1 \mathrm{~ms})$ of high energy photons. Photons with energies up to $40 \mathrm{MeV}$ have been reported [2] recently, which make the TGF the most energetic natural photon phenomenon that exists on Earth. Although we know that TGFs are produced below $20 \mathrm{~km}$ altitudes [3],[4] and related to the lightning discharges, there are many open questions related to their production mechanism and their importance and impact on the Earth's electric circuit. ASIM is designed to address these open questions.

\author{
Carl Budtz-Jørgensen, Irfan Kuvvetli \\ Technical University of Denmark \\ National Space Institute \\ Copenhagen, Denmark
}

The ASIM payload consists of two main scientific instruments. As shown in Fig. 1, the first one is Modular Multispectral Imaging Array (MMIA) composed of 2 cameras and 2 photometers and the second one is MXGS [5-10] composed of 2 detector layers and its readout electronics (see Fig. 2). The ASIM payload is designed and built in collaboration between the Danish Technological University (DTU), University of Bergen (UB), University of Valencia (UV) and Polish Academy of Science (PAS).

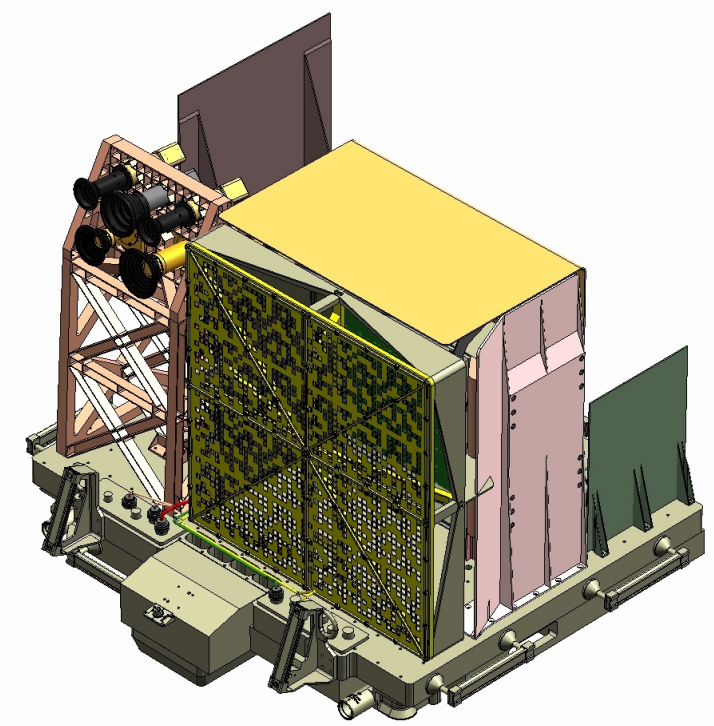

Figure 1. The nadir-viewing assembly (MMIA) of 2 cameras + 2 photometers + MXGS (image credit: DTU).

The focus of this paper is the MXGS instrument, which contains two layers, one pixelated ( $>16000$ pixels) Cadmium Zinc Telluride (CZT) layer, $1024 \mathrm{~cm}^{2}$ in area and $0.5 \mathrm{~cm}$ thick and a Bismuth Germanate Oxide (BGO) layer, $900 \mathrm{~cm}^{2}$ in area and $3.2 \mathrm{~cm}$ thick. The CZT (semiconductor) layer will detect photons in the energy range $15-400 \mathrm{keV}$, while the BGO (scintillator) layer can detect photons up to $20 \mathrm{MeV}$. A coded mask with material that will stop about $50 \%$ of the photons below $200 \mathrm{keV}$ will allow for imaging the low energy photons. Hence, MXGS will be the first instrument to routinely image TGFs and will have 10 times better sensitivity compared to what has been flown to date. 
This paper describes the electronics of the BGO layer and is divided into 6 sections. Following the introduction in section I, an overview of the BGO detector assembly units is presented in section II. In section III, the front-end electronics details are presented, signal processing details are described in section IV, measurement results are discussed in section $\mathrm{V}$ and finally conclusion in section VI.

\section{BGO DETECTOR ASSEMBLY UNITS}

The BGO detector layer consists of four BGO-Detector Assembly Units (BGO-DAU), which again consists of three BGO crystals with corresponding photomultiplier tubes (PMTs) in addition to the readout electronics. Each BGO-DAU is a single electrical unit. The four BGO-DAUs will be mounted on a mechanical support structure and constitute the BGO detector layer with 12 BGO-PMT modules. The BGO detector layer will be constructed as a separate layer in the MXGS system with its own mechanical support, communication links, and power supply inlet. It will be placed underneath the CZT detector layer, thus detecting the radiation which passes through the first layer as depicted in Fig. 2. The performance objectives for the BGO detector layer are given in Table I.

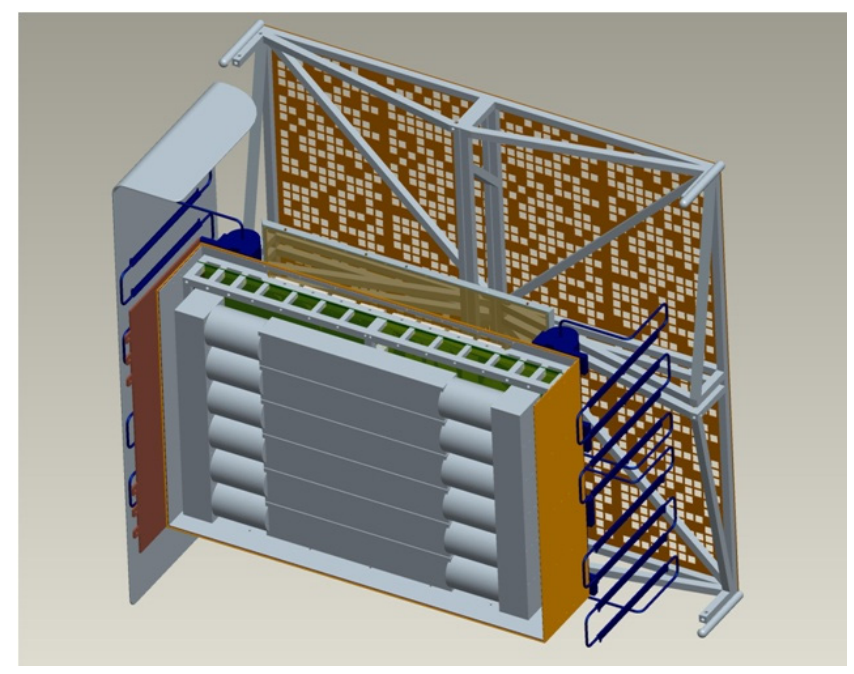

Figure 2. The MXGS seen from behind showing the BGO detector layer in grey and the CZT layer in green (image credit: UV).

TABLE I. SPECIFICATION OF PERFORMANCE PARAMETERS FOR THE BGO DETECTOR LAYER

\begin{tabular}{|c|c|}
\hline Description & Value \\
\hline Energy range & $0.2-20 \mathrm{MeV}$ \\
\hline Energy resolution & $15 \% @ 662 \mathrm{keV}$ \\
\hline Detection efficiency & $>60 \%$ above $1 \mathrm{MeV}$ \\
\hline Relative time accuracy & Better than $10 \mu \mathrm{s}$ \\
\hline Burst rate capability & $650 \mathrm{cts} / \mathrm{ms}$ \\
\hline Instrument background & $2 \mathrm{cts} / \mathrm{ms}(0.2-20 \mathrm{MeV})$ \\
\hline
\end{tabular}

\section{FRONT-END ELECTRONICS FOR BGO DETECTOR LAYER}

Details of the front-end electronics for the BGO detector layer are shown in Fig. 3. To reduce the power consumption and to increase the reliability, component count is optimized and kept as low as possible. There is only one analog signal path per BGO crystal, namely the raw data from the photomultiplier tubes (PMTs). This raw data is pre-amplified, digitized, and processed via a FPGA-based DSP.

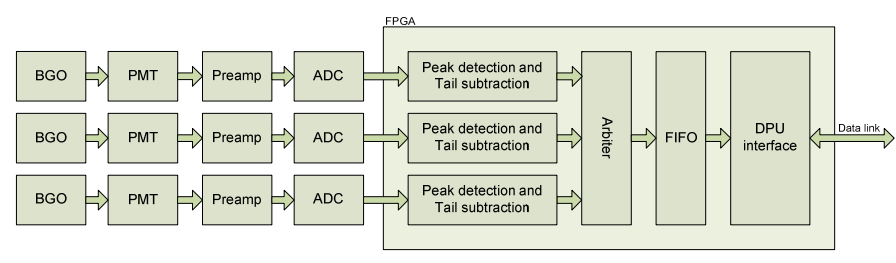

Figure 3. Front-end electronics details for BGO detector layer.

The requirements for the rate capability of the detector are such that pulses are expected to overlap, making it necessary to subtract the tail of previous pulse to obtain the correct amplitude. Sampled data from the Analog-to-Digital Converters (ADCs) is analyzed by a module, which performs offset subtraction and peak detection.

Upon the detection of a pulse, the peak amplitude is embedded in a data packet together with a $1 \mu$ s resolution timetag and various flag bits. The data packets will then be transmitted to the Data Processing Unit (DPU) over an $18 \mathrm{Mbps}$ data/strobe encoded differential serial link. The data packets are 48 bits long, and contain some unused fields for compatibility with the data format from the CZT-layer. Taking into account start and stop bits, each link is able to communicate $330 \mathrm{k}$ packets per second. The DPU interface contains a two-way data link, giving the DPU the ability to configure certain aspects of the DAU, such as enabling or disabling the ADCs, and setting the peak detection threshold. It is also possible to configure a continuous sample mode, where raw sample data is fed directly to the FIFO, then downloaded to the DPU block by block for purposes of diagnostics of the analog system.

\section{A. Analog readout system}

The analog readout system is illustrated in Fig. 4. The current pulse presented on the anode of the PMT is converted to a voltage by resistor $R a$. The signal is then low-pass filtered with a corner frequency of $20 \mathrm{MHz}$ to remove the highfrequency components before the preamp. A high bandwidth is not needed for this application since the time resolution is relatively coarse. The preamp stage is based on the AD844S from Analog Devices, which is configured as a simple voltage amplifier. The output is then converted to a differential signal before the RHF1201 12 bit ADC which is capable of running at high speeds with low power consumption. Sampling rate of the $\mathrm{ADC}$ is set at $36 \mathrm{Msps}$, as this is the frequency of the main oscillator in the system. The ADC driver is an AD8138.

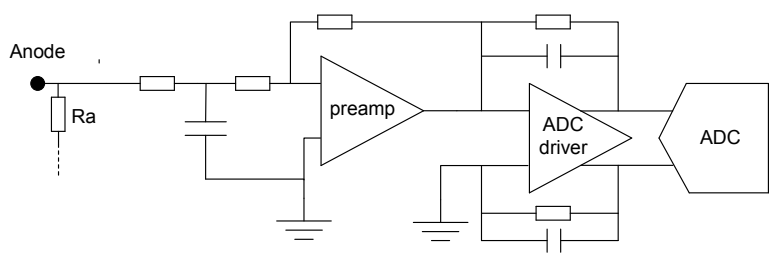

Figure 4. Analog readout system. 
As the pulse shaping is done after digitizing the signal, component count in the proposed analog readout system is far less than the conventional analog readout systems [11],[12]. A PMT pulse, as read by the ADC, is shown in Fig. 5. The area under the pulse represents the energy deposited by the detected gamma ray, which is also proportional to the pulse height.

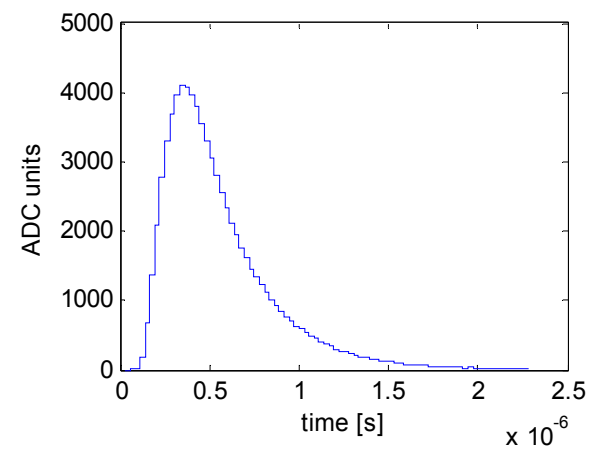

Figure 5. Example PMT pulse as read by the ADC.

\section{Signal Processing For BGO Detector LAYER}

The pulse-processing scheme is depicted in Fig. 6.

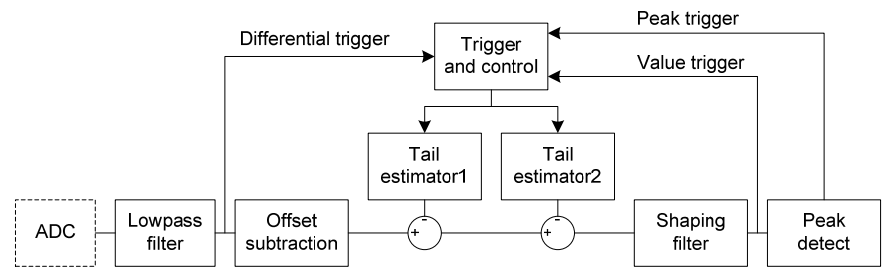

Figure 6. Pulse processing scheme.

First, the raw ADC data is low-pass filtered by a 4-tap running average filter to reduce high frequency noise, which would otherwise disturb the triggering algorithm and reduce the energy resolution. The filter (see Fig. 7) is on an inverted form, lending itself to high-speed operation in the FPGA.

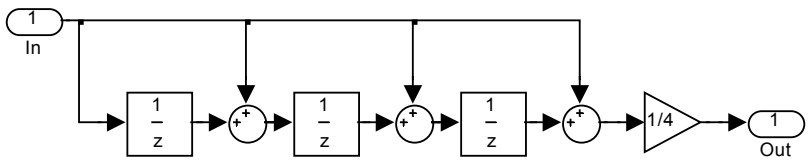

Figure 7. Running average filter.

\section{A. Offset Correction}

Following the low-pass filter is the offset estimation and subtraction, see Fig. 8.

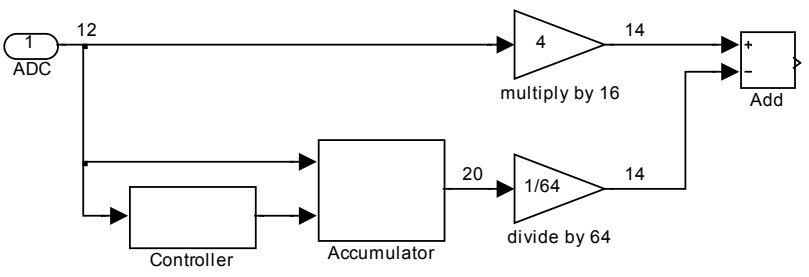

Figure 8. Offset estimation and subtraction.

Unavoidably, there will be an offset in the analog signal before it is digitized. To ensure coherent behaviour of the value threshold, and also to simplify data analysis, the offset is subtracted before the following processing stages. The mean offset value will change with time due to various factors like aging and temperature drifts. The offset will therefore need to be evaluated continuously, and is estimated by performing a time average over a number of consecutive samples. The process is controlled by a state machine that resets the averaging process each time a value is found that deviates from the initial value by more than three standard deviations, as this could indicate a non-systematic effect that would result in an incorrect average. If all samples stay within these bounds, the offset register is updated. The upper bound on the number of samples to average over is dictated by the time it takes to acquire the samples, as well as the size of the accumulator, whereas the lower bound is dictated by the required precision. 256 samples has been found to be a good trade off between the two. This makes it possible to update the offset often, even if pulses are relatively close, while still maintaining sufficient precision. The signal is at the same time up scaled to 14 bits to get some headroom for further processing.

\section{B. Trigger and Control System}

The trigger and control system is controlled by a Concurrent State Machine (CSM). Before the trigger system is activated, the system goes through a start-up procedure, which finds the initial offset value after a delay to allow the analog systems to stabilize. When this is completed, the idle state is entered from where the trigger system is enabled. The trigger system consists of three low-level trigger signals that, when occurring in the correct sequence, will generate a pulse trigger indicating that an event has occurred. The first trigger that is required is the differential trigger. The differential trigger is asserted whenever the difference between two consecutive samples is higher than a certain threshold. The differential trigger is required to be asserted for at least three consecutive clock periods, lest the CSM will return to the idle state. If only a single differential trigger was required, it would trigger on noise quite often, unless the threshold was very high, in which case it would miss many valid triggers. This approach is therefore used to achieve both noise immunity and high sensitivity. The next stage is the value trigger. The CSM will wait for 12 clock cycles to see if the pulse value exceeds the programmable threshold value. If this happens, the system will wait until a peak is found, i.e. it starts to decrease again. This will cause a pulse trigger that will output a time-tagged energy reading.

\section{Dead Time and Rate Capability}

The dead time of a radiation detector is defined as the time after each event during which the system is not able to record another event if it happens. Due to the uncertainty of the temporal distribution of the TGFs, it is difficult to set an exact requirement on the dead time. However, it should be as small as reasonably possible. Hence, the need for tail cancellation arises. With the tail cancellation active, the detector will ignore further triggers (stay in the idle state) for only 10 clock cycles after a pulse trigger. This constitutes a period from peak to trigger reactivation of about $270 \mathrm{~ns}$. The minimum peak-to peak delay depends on the peaking time of the following pulse, which varies slightly as a function of the amplitude, and is also 
affected by noise. Nominal separation between two detectable pulse peaks is about $500 \mathrm{~ns}$, which gives a single BGO chain the capability of continuous rates of $2 \mathrm{Mcps}$ given periodic pulses.

\section{Tail Estimation}

The pulses generated in the BGO have a rise-time in the order of a few ns, and the decay time is well approximated by an exponential function, with a time constant of about $300 \mathrm{~ns}$ at $20^{\circ} \mathrm{C}$, although with a strong temperature dependence.

Fig. 9 shows two pulses occurring within a short time, resulting in the second one having distorted amplitude. The actual energy of the second pulse is illustrated by the dotted vertical line. The estimated tail of the first pulse is also shown. The purpose of the tail estimators is to estimate and subtract these pulse tails in real-time, shortening the pulses, and thereby reducing the dead-time of the detector.

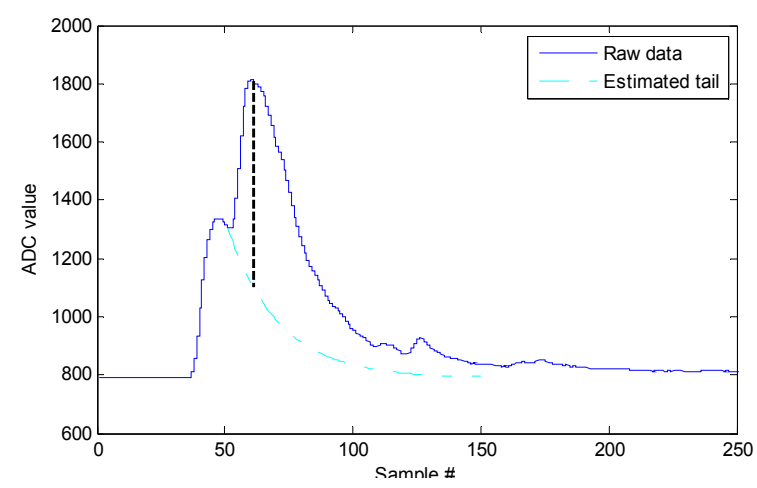

Figure 9. Pulse pileup with tail estimation.

\section{MEASUREMENT Results}

Fig. 10 shows calibration curve and the energy spectrum of ${ }^{137} \mathrm{Cs}$ and ${ }^{60} \mathrm{Co}$ standard gamma-ray sources with the proposed front-end electronics. As can be observed, $1332 \mathrm{keV}$ line is clearly resolved with the $1173 \mathrm{keV}$ line for ${ }^{60} \mathrm{Co}$. The energy resolution of gamma-ray line at $667 \mathrm{keV}$ is $116.5 \mathrm{keV}$ FWHM $(17.6 \%)$. Measured resolution is close to the required specifications and it is expected to be improved in the flight version.

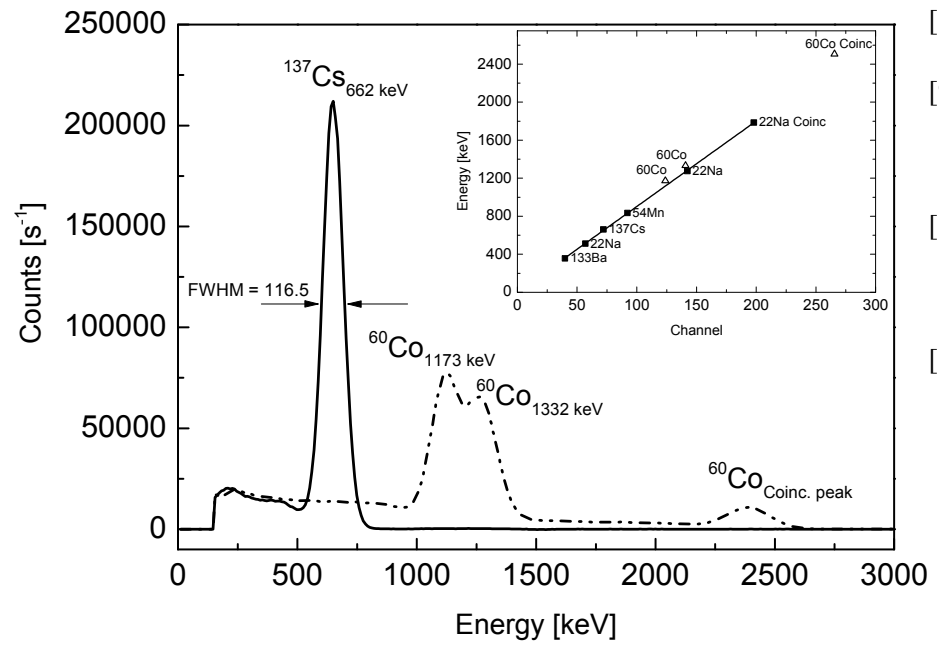

Figure 10. Energy spectrum of ${ }^{137} \mathrm{Cs}$ and ${ }^{60} \mathrm{Co}$ standard gamma-ray sources.

\section{CONCLUSION}

The digital pulse processing scheme used in the MXGS BGO detector gives it a significantly higher rate capability than what has been achieved in other instruments used in the study of terrestrial gamma flashes. This will enable the phenomenon to be studied in greater detail than what was previously possible.

The front-end electronics for the BGO detector layer in MXGS system also uses fewer components compared to conventional analog front-ends for BGO detectors, thereby increasing its reliability and projected lifetime in the harsh space environment. It is expected that ASIM/MXGS will see about 1000 TGFs a year (ten times RHESSI and 100 times BATSE) and for each TGF we will have about 300 counts (ten times RHESSI).

\section{ACKNOWLEDGEMENTS}

This project is supported by European Space Agency as a subsub-contract between DTU and UB, to the contract 4000101107/10/NL/BJ between ESTEC and TERMA Ref. and by Norwegian Research Council under contract 184790/V30.

\section{REFERENCES}

[1] Fishman, G. J., et al. (1994), Discovery of intense gamma-ray flashes of atmospheric origin, Science, 264(5163), 1313-1316.

[2] Marisaldi, M. et al. (2010), Detection of Terrestrial Gamma-Ray Flashes up to $40 \mathrm{MeV}$ by the AGILE satellite, J. Geophys. Res, 115, A00E13, doi:10.1029/2009JA014502.

[3] Gjesteland, T, N. Østgaard, J. Stadsnes, P. H. Connell, G. J. Fishman, (2010) Effects of deadtime losses on Terrestrial Gamma ray Flash measurements done by the Burst And Transient Source Experiment, J. Geophys. Res.,115, A00E21, doi: 10.1029/2009JA014754.

[4] Carlson, B. E., N. G. Lehtinen, and U. S. Inan (2007), Constraints on terrestrial gamma ray flash production from satellite observation, Geophys. Res. Lett., 34, L08,809, doi:10.1029/2006GL029229.

[5] ASIM Topical Team meeting, http://www.dsri.dk/atmosphere/asim/tt1/tt1.html (last accessed, Nov. 2011).

[6] Thomsen, P. L., "ASIM Payload System Overview," ASIM Topical Team Meeting-1, ESA/ESTEC, June 26-27, 2006.

[7] Neubert, T., et.al., "The Atmosphere-Space Interactions Monitor (ASIM) for the International Space Station,” ILWS (International Living With a Star) Workshop 2006, Goa, India, Feb. 19-20, 2006.

[8] Reibaldi, G.,et.al., "The ESA Payloads for Columbus- A bridge between the ISS and exploration," ESA Bulletin, No 122, May 2005, pp. 60-70.

[9] Neubert, T., "The Atmosphere-Space Interactions Monitor (ASIM) for the International Space Station," Workshop on Coupling of Thunderstorms and Lightning Discharges to Near-Earth Space, June 2327, 2008, University of Corsica, Corte, France.

[10] Zhang, N., et al, "Anode Position and Last Dynode Timing Circuits for Dual-Layer BGO Scintillator With PS-PMT Based Modular PET Detectors," IEEE Transactions on Nuclear Science, Vol. 49, No. 5, pp. 2203-2207, 2002.

[11] Meegan, C., et al, "THE FERMI GAMMA-RAY BURST MONITOR," The Astrophysical Journal, Vol. 702, Iss. 1, pp. 791-804, 2009. 